\title{
Singlet state formation and its impact on the magnetic structure in the tetramer system $\mathrm{SeCuO}_{3}$
}

\author{
Tonči Cvitanić, ${ }^{1}$ Vinko Šurija, ${ }^{2}$ Krunoslav Prša, ${ }^{3}$ Oksana Zaharko, ${ }^{4}$ Ivan Kupčić, ${ }^{1}$ Peter Babkevich, ${ }^{3}$ Matthias Frontzek, \\ Miroslav Požek, ${ }^{1}$ Helmuth Berger, ${ }^{3}$ Arnaud Magrez, ${ }^{3}$ Henrik M. Rønnow, ${ }^{3}$ Mihael S. Grbić, ${ }^{1, *}$ and Ivica Živkoviće, ${ }^{2, \dagger}$ \\ ${ }^{1}$ Department of Physics, Faculty of Science, University of Zagreb, Bijenička 32, HR-10000 Zagreb, Croatia \\ ${ }^{2}$ Institute of Physics, Bijenička 46, HR-10000 Zagreb, Croatia \\ ${ }^{3}$ Institute of Physics, EPFL, CH-1015 Lausanne, Switzerland \\ ${ }^{4}$ Laboratory for Neutron Scattering and Imaging, PSI, CH-5232 Villigen, Switzerland \\ ${ }^{5}$ Neutron Scattering Division, Oak Ridge National Laboratory, Oak Ridge, Tennessee 37831, USA
}

(Received 11 January 2018; revised manuscript received 26 June 2018; published 9 August 2018)

\begin{abstract}
We present an experimental investigation of the magnetic structure in a tetramer system $\mathrm{SeCuO}_{3}$ using neutron powder-diffraction and nuclear resonance techniques. We establish a commensurate antiferromagnetic ordering with a propagation vector $\mathbf{k}=(0,0,1)$. The order parameter follows a critical behavior near $T_{N}=8 \mathrm{~K}$ with a critical exponent $\beta=0.32$ in agreement with a three-dimensional universality class. Evidence is presented that a singlet state starts to form on tetramers at temperatures as high as $200 \mathrm{~K}$, and its signature is preserved within the ordered state through a strong renormalization of the ordered magnetic moment on two nonequivalent copper sites $m_{\mathrm{Cu} 1} \approx 0.35 \mu_{\mathrm{B}}$ and $m_{\mathrm{Cu} 2}<0.8 \mu_{\mathrm{B}}$ at $1.5 \mathrm{~K}$.

DOI: 10.1103/PhysRevB.98.054409
\end{abstract}

\section{INTRODUCTION}

Classical three-dimensional (3D) magnetic systems tend to order in a long-range magnetic structure due to the presence of interaction $J$ between magnetic moments. Their behavior around the ordering temperature is well understood in terms of thermally induced fluctuations. More interesting behavior is found when the system is subject to quantum fluctuations, governed by an external parameter other than temperature. Quantum effects become especially important when the geometrical arrangement of magnetic moments induces frustration in the selection of a unique ground state via competing interactions and/or the effective dimensionality $D$ is reduced to $D<3$. Recent investigations of triangular- and kagome-based systems $[1,2]$ with signatures of a potential quantum spin-liquid phase reveal how quantum effects can completely prevent long-range order from occurring.

The ultimate limit of low-dimensional reduction is reached when a local cluster of spins is separated from other clusters around it. Significant attention has been devoted to singlemolecule magnets with strong single-ion anisotropy governing macroscopic quantum tunneling of magnetization. [3] In these metal-organic compounds the interaction between clusters is negligible due to large separation by organic ligands. On the other hand, in recent years several compounds have been discovered where the intercluster interaction allows the observation of subtle effects that govern the transition from local quantum states towards delocalized spin waves. For instance in $\mathrm{TlCuCl}_{3}$ pressure can be used as a tuning parameter for intercluster interaction, allowing the observation of closing the singlet-triplet gap and the emergence of longrange antiferromagnetic (AFM) order [4]. In other compounds

\footnotetext{
*mgrbic@phy.hr

†ivica.zivkovic@epfl.ch
}

where the interactions are not easily modified, it remains an outstanding question of how the specific ratio of intra- to intercluster interaction(s) influences magnetic properties of a given compound. This becomes especially interesting when clusters are composed of more than just two AFM-coupled spins, increasing the number of local quantum states. With three spins in the cluster the ground state has a nonzero spin state and a divergent magnetic susceptibility when $T \rightarrow 0$. The four-spin AFM-based clusters, called tetramers, again exhibit a singlet ground state, but now the excited states comprise additional singlet states, three triplets and a quintet. Their relative order is determined by the geometry of the cluster and the relative strength of intracluster interactions [5].

Several compounds have been reported that adhere to the weakly coupled tetramer model. A tetrahedron configuration of $S=1 / 2$ spins have been found in $\mathrm{Cu}_{2} \mathrm{Te}_{2} \mathrm{O}_{5} \mathrm{Cl}_{2}$ and $\mathrm{Cu}_{2} \mathrm{Te}_{2} \mathrm{O}_{5} \mathrm{Br}_{2}$ with intracluster interactions only twice larger than intercluster ones [6]. A diamond-shape cluster has been found in $\mathrm{Cu}_{2} \mathrm{PO}_{4} \mathrm{OH}$ with intracluster interaction around $140 \mathrm{~K}$ and without long-range order down to $2 \mathrm{~K}$ [7]. A linear tetramer system has been found in $\mathrm{Cu}_{2} \mathrm{CdB}_{2} \mathrm{O}_{6}$ [8], $\mathrm{SeCuO}_{3}$ [9], and $\mathrm{CuInVO}_{5}$ [10], all showing strong intracluster interactions of 100-300 K with long-range order occurring below $10 \mathrm{~K}$, suggesting intercluster interactions two orders of magnitude smaller.

In this article we focus our attention on $\mathrm{SeCuO}_{3}$ and the peculiarities of its magnetic ground state. $\mathrm{SeCuO}_{3}$ crystallizes in a monoclinic unit cell with the space-group $P 2_{1} / n$ as reported in Ref. [11]. It has two crystallographically inequivalent copper sites, $\mathrm{Cu} 1$ and $\mathrm{Cu} 2$. Each copper is surrounded by six oxygen atoms which form elongated octahedrons: The four nearest oxygen ions (distance around $1.9 \AA$ ) form $\mathrm{CuO}_{4}$ plaquettes, with apical oxygens further away $(2.4 \AA)$. This crystal configuration places the $\left(d_{x^{2}-y^{2}}\right)$-dominated orbital highest in energy with a single magnetically active electron 

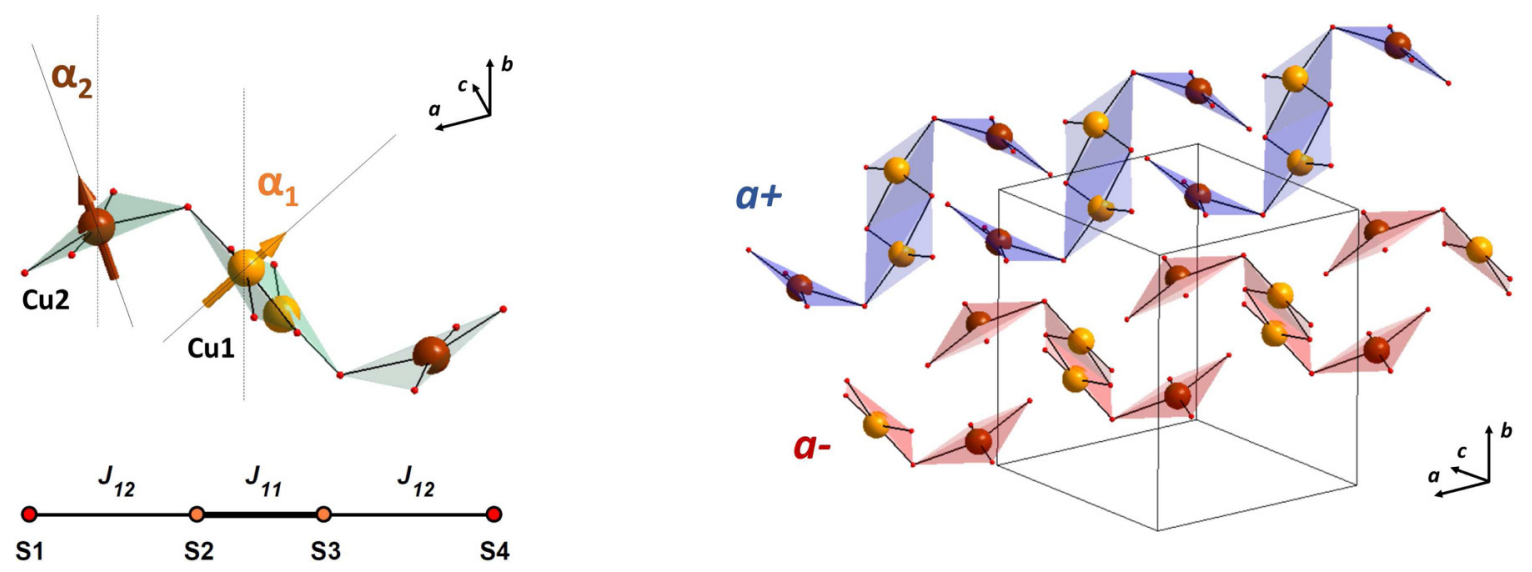

FIG. 1. Left: A single tetramer with shaded $\mathrm{CuO}_{4}$ plaquettes. The direction of $V_{z z}$ is drawn for $\mathrm{Cu} 1$ and $\mathrm{Cu} 2$ sites together with angles that they close with the $b$ axis $\left(\alpha_{1}=48^{\circ}, \alpha_{2}=25^{\circ}\right)$. Below a schematic of spins and exchange interactions between them [see Eq. (1)]. Right: two chains of tetramers (blue and green) are oriented along the $a$ axis with a different sense of direction ( $a+$ and $a-$, respectively). They form interpenetrating lattices with red chains propagating in the middle of the unit cell, whereas the blue ones-along its edges. Only one blue chain is shown for clarity. Selenium atoms are omitted.

[9]. Each Cu1 site is connected via two oxygens to another $\mathrm{Cu} 1$ site (interaction $J_{11}$ ) and with a single oxygen to the $\mathrm{Cu} 2$ site (interaction $J_{12}$ ), effectively forming a $\mathrm{Cu} 2-\mathrm{Cu} 1-\mathrm{Cu} 1-\mathrm{Cu} 2$ linear tetramer (Fig. 1).

Each tetramer has a shape of a "seahorse," forming a onedimensional chain of tetramers along the $a$ axis with a "headover-tail" coupling between the neighbors (weak intertetramer $J_{22}$ interaction between two $\mathrm{Cu} 2$ moments mediated by an almost perpendicular $\mathrm{Cu}-\mathrm{O}-\mathrm{Cu}$ bridge). There are two chains, one running along the " $a+$ " direction and one along the " $a-$ " direction (in Fig. 1 these chains are shown by blue and red, respectively). Tetramers from different chains are symmetry related by the $180^{\circ}$ rotation, followed by the [100] translation. The coupling across the chains is mediated through the network of $\mathrm{SeO}_{3}$ tetrahedra (not shown).

The Hamiltonian of a spin tetramer is expressed through the Heisenberg interactions,

$$
\mathcal{H}=J_{11} \mathbf{S}_{\mathbf{2}} \cdot \mathbf{S}_{\mathbf{3}}+J_{12}\left(\mathbf{S}_{\mathbf{1}} \cdot \mathbf{S}_{\mathbf{2}}+\mathbf{S}_{\mathbf{3}} \cdot \mathbf{S}_{\mathbf{4}}\right) .
$$

Previous results $[9,12]$ showed that magnetic moments in $\mathrm{SeCuO}_{3}$ form isolated tetramers, so the system behaves like a quasi-zero-dimensional antiferromagnet. The tetramer Hamiltonian [Eq. (1)] was used to explain experimental results down to $T \approx 90 \mathrm{~K}$, determining $J_{11}$ and $J_{12}$ to be approximately 225 and $160 \mathrm{~K}$, respectively. The system remains disordered down to $T_{N}=8 \mathrm{~K}$ where a 3D AFM order sets in. The magnetically ordered ground state emerges when the singlet ground state hybridizes with the excited states due to mixing by intertetramer interactions and/or anisotropy terms. No clear model exists to explain the experimental results in the intermediate phase $(8-90 \mathrm{~K})$. The tetramer model is not able to reproduce the steep increase in magnetic susceptibility, but a broad maximum around $20 \mathrm{~K}$ suggests low-dimensional behavior. An interesting aspect has been revealed by electron spin resonance (ESR) and torque magnetometry [12] where a strong rotation of magnetic axes in the intermediate-temperature range was observed. Recent magnetization measurements at a high magnetic field, ESR, and ${ }^{77} \mathrm{Se}$ nuclear magnetic resonance (NMR) reported a magnetic anomaly in the ordered phase below
$T=6 \mathrm{~K}$ which supposedly occurs due to a spin reorientation (Ref. [13]).

In this paper we address the aspects of the magnetic structure within the ordered state using neutron diffraction and ${ }^{63,65} \mathrm{Cu}$ nuclear quadrupolar resonance (NQR) measurements. We found no trace of the anomaly or spin reorientation reported in Ref. [13] and present evidence of the Cu1-Cu1 spin singlet formation below $T \approx 200 \mathrm{~K}$.

\section{EXPERIMENTAL DETAILS}

Single crystals of $\mathrm{SeCuO}_{3}$ were grown by a standard chemical vapor phase method. Mixture of analytical grade purity $\mathrm{CuO}$ and $\mathrm{SeO}_{2}$ powders in a molar ratio of $4: 3$ was sealed in the quartz tubes with electronic grade $\mathrm{HCl}$ as the transport gas for the crystal growth. The ampules were then placed horizontally into a tubular two-zone furnace and heated very slowly by $50^{\circ} \mathrm{C} / \mathrm{h}$ to $500^{\circ} \mathrm{C}$. The optimum temperatures at the source and deposition zones for the growth of single crystals were $550^{\circ} \mathrm{C}$ and $450^{\circ} \mathrm{C}$, respectively, and after four weeks many green $\mathrm{SeCuO}_{3}$ crystals were obtained. The phase purity was verified using x-ray powder diffraction.

Neutron powder-diffraction patterns were collected on the Cold Neutron Powder Diffractometer at SINQ, Paul Scherrer Institute, Switzerland. The 4.2-g $\mathrm{SeCuO}_{3}$ sample was sealed in an 8-mm diameter cylindrical vanadium can under helium and cooled down in the Orange ILL-type cryostat. A neutron wavelength of $\lambda=2.46 \AA$ was used, and about $6 \mathrm{~h}$ was needed for one temperature point within the (1.5-15)-K range. The data were analyzed and refined using the FULLPROF suite [14].

To get a better insight into the local spin behavior, NMR and NQR techniques were used. These techniques locally probe interactions with observed nuclei, being sensitive to an external magnetic field and to an electric-field gradient (EFG) on the nuclei. Since the magnetically active electrons reside on the $\mathrm{Cu}$ sites, ${ }^{63,65} \mathrm{Cu} \mathrm{NMR} / \mathrm{NQR}$ was chosen for this experiment as it provides direct insight into the local spin behavior. 

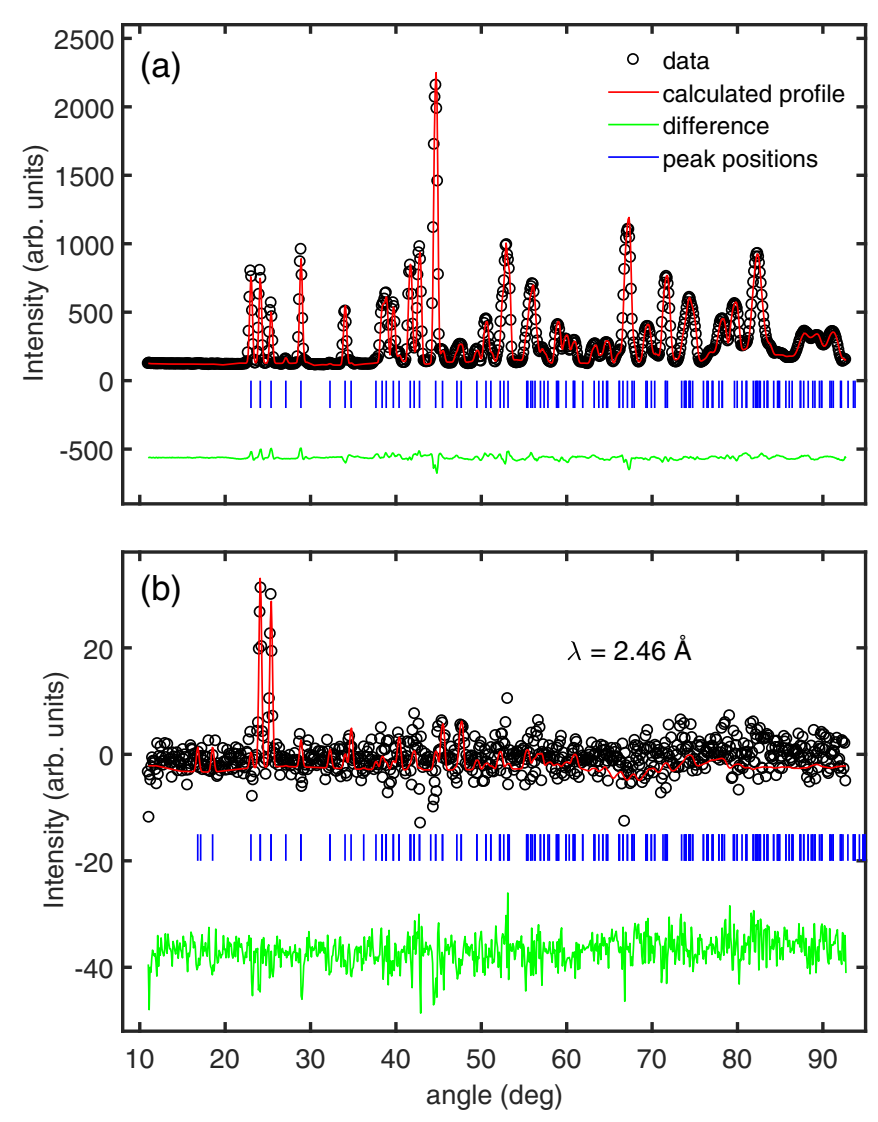

FIG. 2. (a) Neutron powder-diffraction pattern of $\mathrm{SeCuO}_{3}$ at $15 \mathrm{~K}$. The solid red line is a calculated pattern for $\mathrm{SeCuO}_{3}$ using the space-group $P 2_{1} / n$, whereas the solid green line indicates the difference between calculated and observed intensities. Vertical bars indicate calculated positions of Bragg reflections. (b) Magnetic diffraction pattern at $1.5 \mathrm{~K}$ after subtracting pattern at $15 \mathrm{~K}$. Only two significant peaks are observed, corresponding to pairs $(011) /(01 \overline{1})$ and $(110) /(1 \overline{1} 0)$. The solid red line represents a calculated intensity using the $\Gamma_{4}$ irreducible representation (IR).

The NMR/NQR experiment was conducted in a superconducting Oxford Instruments variable-field magnet. The sample was a single crystal $\left(5 \times 1 \times 1 \mathrm{~mm}^{3}\right)$ with the longest axis along the $b$ axis. A copper coil was tightly wound around the longest axis of the sample, which was mounted on a single axis rotator with the axis of rotation within $5^{\circ}$ from the $b$ axis. Spectra were acquired with a Tecmag Apollo spectrometer by the standard Hahn echo sequence $\pi / 2-\tau-\pi$, followed by the Fourier transform of the echo signal. The duration of the $\pi / 2$ pulse was $1.4 \mu \mathrm{s}$, and the time between the pulses was varied from $\tau=15 \mu \mathrm{s}$ at low temperatures to $\tau=3 \mu \mathrm{s}$ at $T=200 \mathrm{~K}$ and above. Multiple spectra were added together to obtain a broadband spectra by the usual variable offset cumulative spectroscopy method.

\section{EXPERIMENTAL RESULTS}

\section{A. Neutron powder diffraction}

Figure 2(a) shows the powder neutron-diffraction pattern of $\mathrm{SeCuO}_{3}$ measured at $15 \mathrm{~K}$. Refinement was performed against the model presented in Ref. [9]. The agreement
TABLE I. Basis functions of the irreducible representations for the $\mathrm{Cu} 1$ and $\mathrm{Cu} 2$ sites (using the same notation as in Ref. [9]) in the space-group $P 2_{1} / n$ with the propagation vector $\mathbf{k}=(0,0,1)$ for $\mathrm{SeCuO}_{3}$ obtained from the representational analysis. Each crystallographic $\mathrm{Cu}$ site has four $\mathrm{Cu}$ atoms: $\mathrm{Cu}^{I}:(x, y, z), \mathrm{Cu}^{I I}:(-x+$ $1 / 2, y+1 / 2,-z+1 / 2), \mathrm{Cu}^{I I}:(-x,-y,-z)$, and $\mathrm{Cu}^{I V}:(x+$ $1 / 2,-y+1 / 2, z+1 / 2)$. Since all basis functions of these IRs contain only real components and all have only diagonal elements, only the diagonal terms are given in table below.

\begin{tabular}{|c|c|c|c|c|c|}
\hline IRs & Site: & $\mathrm{Cu}^{I}$ & $\mathrm{Cu}^{I I}$ & $\mathrm{Cu}^{I I I}$ & $\mathrm{Cu}^{I V}$ \\
\hline$\Gamma_{1}$ & & $(1,1,1)$ & $(-1,1,-1)$ & $(1,1,1)$ & $(-1,1,-1)$ \\
\hline$\Gamma_{2}$ & & $(1,1,1)$ & $(-1,1,-1)$ & $(-1,-1,-1)$ & $(1,-1,1)$ \\
\hline$\Gamma_{3}$ & & $(1,1,1)$ & $(1,-1,1)$ & $(1,1,1)$ & $(1,-1,1)$ \\
\hline$\Gamma_{4}$ & & $(1,1,1)$ & $(1,-1,1)$ & $(-1,-1,-1)$ & $(-1,1,-1)$ \\
\hline
\end{tabular}

between the published and the refined unit-cell parameters $a=7.6989(2), b=8.2126(2), c=8.4856(2) \AA$, and $\beta=$ $99.178(2)^{\circ}$ is very good.

The difference between the patterns collected at 15 and $1.5 \mathrm{~K}$ is shown in Fig. 2(b). The difference pattern contains only two magnetic reflections since the structural changes between the two temperatures are negligible. The magnetic peaks are found on top of nuclear ones making separation of the purely magnetic signal challenging.

Using the K-SEARCH program from the FULLPROF suite we determined the magnetic propagation vector $\mathbf{k}=(0,0,1)$. This reveals commensurate antiferromagnetic ordering. To constrain the possible magnetic structures, we have performed irreducible representation analysis using the program BASIREPS from the FULLPROF suite. The symmetry analysis for the propagation vector $\mathbf{k}=(0,0,1)$ and space-group $P 2_{1} / n$ yielded only four nonzero one-dimensional $\left(\Gamma^{1}\right)$ IRs, each with the multiplicity of 3 . The magnetic representation $\Gamma_{\text {mag }}$ is thus composed of

$$
\Gamma_{\mathrm{mag}}=3 \Gamma_{1}+3 \Gamma_{2}+3 \Gamma_{3}+3 \Gamma_{4},
$$

where superscript 1 is omitted for clarity. The basis vectors for these IRs are listed in Table I.

$\Gamma_{1}$ and $\Gamma_{3}$ suggest a ferromagnetic coupling between $\mathrm{Cu} 1$ moments and predict reflections which are not observed [(101)

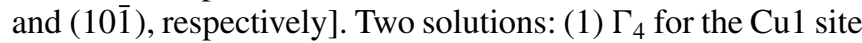
and $\Gamma_{2}$ for the $\mathrm{Cu} 2$ site and (2) $\Gamma_{4}$ IR for both sites, gave equally good agreement with the data. As the transition from the paramagnetic state to the magnetically ordered state is of the second order, we conclude that both copper sites must order with the same $\Gamma_{4}$ IR.

With only two magnetic peaks present in the powderdiffraction pattern, we are at the moment not able to provide all the details of the magnetic structure [15]. Several possible spin configurations result in a very similar diffraction pattern, and the red solid line in Fig. 2(b) represents a diffraction from a spin configuration where the orientation of $\mathrm{Cu} 1$ spins lie within the $\mathrm{CuO}_{4}$ plaquette as suggested by NQR results (see below). In this case we obtain a very plausible configuration where also $\mathrm{Cu} 2$ spins lie within their $\mathrm{CuO}_{4}$ plaquette. Still, the exact orientation within plaquettes is not uniquely determined. 


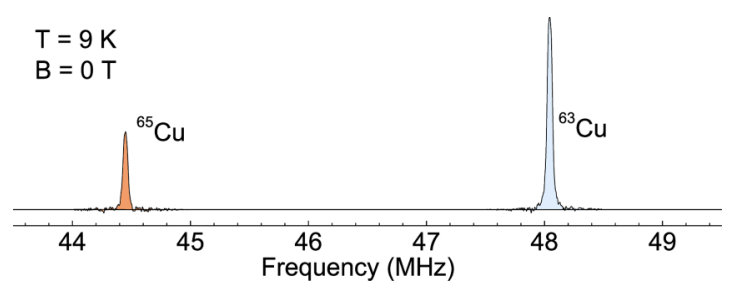

FIG. 3. NQR spectrum at $T=9 \mathrm{~K}$, above antiferromagnetic transition. Two different $\mathrm{Cu}$ isotopes are labeled and colored accordingly: red for ${ }^{65} \mathrm{Cu}$ and blue for ${ }^{63} \mathrm{Cu}$. From the crystal structure, we should see two copper sites ( $\mathrm{Cu} 1$ and $\mathrm{Cu} 2)$, so the number of lines should be four (two for each isotope). Missing lines imply that one copper site has a fast relaxation rate, and it is not visible by NQR/NMR.

Nevertheless, several key aspects can be inferred: The $\Gamma_{4}$ IR indicates an antiparallel arrangement of spins on the equivalent copper sites $\uparrow_{\mathrm{Cu} 1}-\downarrow_{\mathrm{Cu} 1}$ and $\uparrow_{\mathrm{Cu} 2}-\downarrow_{\mathrm{Cu} 2}$, supporting the hypothesis presented from macroscopic measurements [9]. Furthermore, whichever structure has been attempted, the resulting values of magnetic moments on two copper sites are strongly renormalized. We can put an upper limit for each site $m_{\mathrm{Cu} 1}<0.5 \mu_{\mathrm{B}}$ and $m_{\mathrm{Cu} 2}<0.8 \mu_{\mathrm{B}}$, indicating a strong renormalization from the nominal $1 \mu_{\mathrm{B}}$ value. The fact that the inner copper sites $(\mathrm{Cu} 1)$ have smaller ordered moments than the outer ones $(\mathrm{Cu} 2)$ supports the initial model based from the susceptibility analysis $[9,12]$ where the $J_{11}$ coupling is notably stronger than $J_{12}$. This induces stronger quantum fluctuations from the singlet state.

\section{B. Nuclear quadrupolar resonance-paramagnetic phase}

The NMR and NQR methods probe nuclear spins and their coupling to the local magnetic field and EFG, respectively. EFG is a tensor defined as $V_{i j}=\partial V / \partial x_{i} \partial x_{j}$, usually expressed with principal axes following the standard convention $\left|V_{z z}\right|>$ $\left|V_{y y}\right|>\left|V_{x x}\right|$. Within $\mathrm{SeCuO}_{3}$, copper nuclei have nonvanishing EFGs that give rise to a NQR signal.

Above $T_{N}$ the NQR spectra show two distinct lines that correspond to two copper isotopes ${ }^{65} \mathrm{Cu}$ and ${ }^{63} \mathrm{Cu}$, shown in Fig. 3. The lines can be fit to a Gaussian function with FWHM of $40 \mathrm{kHz}$. The linewidth is constant in the temperature range from $T=9$ to $T=260 \mathrm{~K}$, indicating no onset of short-range order.

With two inequivalent crystallographic sites ( $\mathrm{Cu} 1$ and $\mathrm{Cu} 2)$, one would expect different EFG values for each of them so that four NQR lines should be visible, two for each isotope. A second pair of lines was searched for in a broad frequency range from 16 to $60 \mathrm{MHz}$. Lack of the second pair of lines, even with short interpulse time $(\tau=3 \mu \mathrm{s})$, implies that the relaxation rate of one copper site is too fast to be detected by NQR.

To determine which $\mathrm{Cu}$ site is observed by NQR, we measured an angle dependence of the Zeeman-perturbed NQR of $\mathrm{SeCuO}_{3}$ [Fig. 4(a)] above $T_{N}$. By applying a small external field, the single NQR line splits into four lines, whose positions depend on the angle of the external magnetic field with respect to the principal axis $V_{z z}$. The sample rotation was performed around the $b$ axis, whereas the magnetic field was applied within the $a c$ plane. (a)
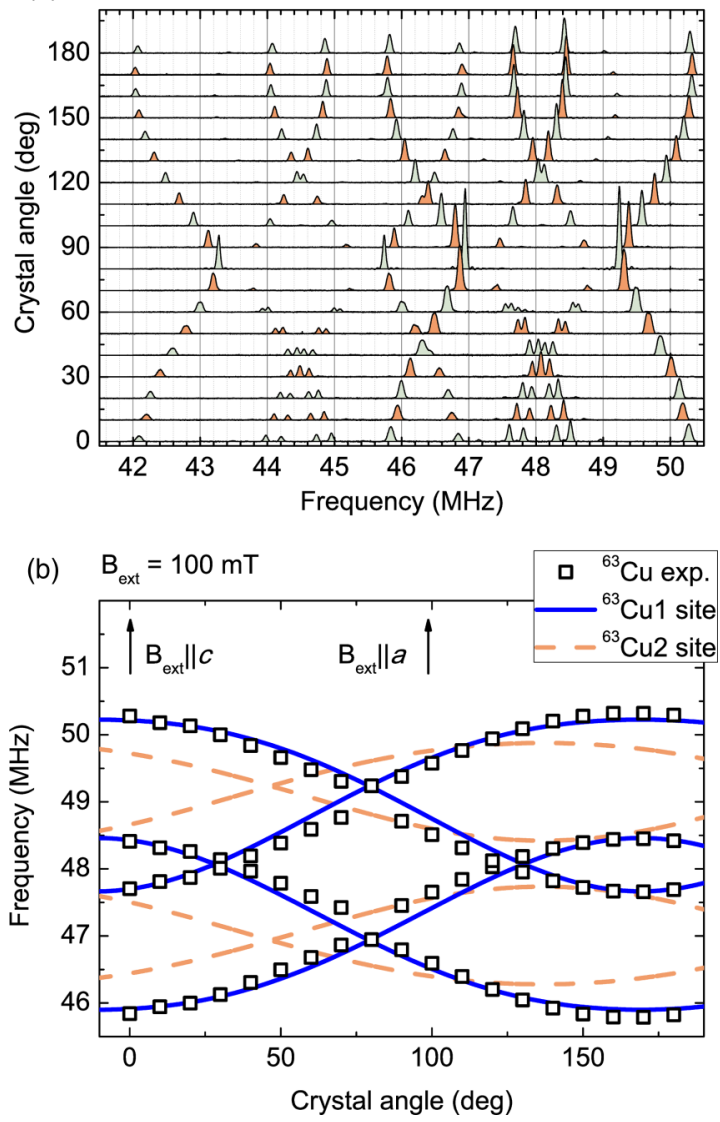

FIG. 4. (a) Rotated field-perturbed NQR spectra. $B=100 \mathrm{mT}$, $T=19 \mathrm{~K}$. Eight lines can be easily distinguished (four per each isotope). Small additional line splitting (near $0^{\circ}$ ) arises from a small misalignment: the $b$ axis of the sample is not completely perpendicular to the external magnetic field. (b) Line position of ${ }^{63} \mathrm{Cu}$ at different sample orientations (white squares) from (a), $T=19 \mathrm{~K}$. The crystal angle is the angle between the crystallographic $c$ axis and the external magnetic field $\left(B_{\text {ext }}=100 \mathrm{mT}\right.$ ). A simulation of line position (see the text) for two copper sites is shown: $\mathrm{Cu} 1$ (solid blue line) and $\mathrm{Cu} 2$ site (dashed green lines). The $\mathrm{Cu} 1$ site better fits the observed data since the $\mathrm{Cu} 2$ site lacks crossing of the inner lines (at $30^{\circ}$ and $130^{\circ}$ ). Only two lines are present at $80^{\circ}$, implying asymmetry parameter $\eta=0$ (see the text).

The complete NMR/NQR Hamiltonian [16] is as follows:

$$
\begin{aligned}
\mathcal{H}= & -\gamma_{n} \hbar B I_{z} \cos \vartheta+\frac{e Q V_{z z}}{4 I(2 I-1)} \\
& \times\left[3 I_{z}^{2}-I^{2}+\eta\left(I_{x}^{2}-I_{y}^{2}\right)\right],
\end{aligned}
$$

where $\eta=\left(V_{y y}-V_{x x}\right) / V_{z z}$ is the asymmetry parameter, $\vartheta$ is the angle between the local $z$ axis and the local magnetic-field $\boldsymbol{B}, \mathbf{I}$ is the nuclear spin (for copper $I=3 / 2$ ), and $Q$ and $\gamma_{n}$ are the quadrupole moment and the gyromagnetic ratio of the nucleus, respectively. The local symmetry of the copper sites implies that $V_{z z}$ is oriented perpendicular to the plane defined by the four oxygens in each $\mathrm{CuO}_{4}$ plaquette. We have checked our reasoning by calculating the eigenvalues and eigenvectors of the EFG tensors with a complete point-charge model that takes into account both local and lattice contributions $[17,18]$. The results show that the taken directions of the $V_{z z}$ principal axes do not change by more than $\approx 2^{\circ}$, and the calculated EFG 
tensors all have $\eta<0.1$, which is well within the resolution of our experiment.

Numerical diagonalization of the Hamiltonian was used to explain the spectra. Four lines per copper site instead of two arise due to mixing of nuclear spin states that is beyond firstorder perturbation theory. Free parameters are the asymmetry $\eta$ and the angle $\vartheta . V_{z z}$ and $\eta$ are interdependent, and their relation can be deduced from NQR at $B=0 \mathrm{~T}$ (Fig. 3). In that case, the line position is given as $v_{Q}=\frac{e Q V_{z z}}{2} \sqrt{1+\eta^{2} / 3}$.

Since two crystallographic sites $(\mathrm{Cu} 1$ and $\mathrm{Cu} 2)$ will close different $\vartheta$ angles with the magnetic field, consequentially they will have different line positions which allows us to determine which site is visible in the experiment. From the crystal structure it follows that for the Cu1 site $V_{z z}$ forms an angle with the crystal $b$ axis of $\alpha_{1}=48^{\circ}$ and for the $\mathrm{Cu} 2$ site $\alpha_{2}=25^{\circ}$ (see Fig. 1). As the the sample is rotated around the $b$ axis, the angle [ $\vartheta$ in (3)] between the external field (on the $a c$ plane) and $V_{z z}$ varies from $90^{\circ}-48^{\circ}=42^{\circ}$ to $90^{\circ}$ (perpendicular to $V_{z z}$ ) for the $\mathrm{Cu} 1$ site and from $90^{\circ}-$ $25^{\circ}=65^{\circ}$ to $90^{\circ}$ for the $\mathrm{Cu} 2$ site. The calculated line positions for $B=B_{\text {ext }}=100 \mathrm{mT}$, and experimental results for rotation spectra around the crystal $b$ axis are shown in Fig. 4(b). Two distinct features are clearly visible. The crossing of inner lines at angles of $30^{\circ}$ and $130^{\circ}$ implies $\vartheta$ to be equal to the magic angle $\left(54.7^{\circ}\right)$-a well-known feature of the NMR Hamiltonian [19]. Analyzing the crystallographic data and the direction of rotation we find that only the NQR signal of the Cu1 site can reproduce this crossing since the magic angle is within the region $\left(90^{\circ}, 42^{\circ}\right)$ and not for $\mathrm{Cu} 2$ where $\vartheta$ is within the region $\left(90^{\circ}, 65^{\circ}\right)$. Therefore, the observed signal can be designated, based on symmetry arguments, to the $\mathrm{Cu} 1$ site.

The other feature of the NQR spectrum is the existence of a single line at $80^{\circ}$, which is possible only for $\eta=0$. Thus, all parameters of the Hamiltonian are determined.

An opposite designation of sites would be possible only if the direction of the $V_{z z}$ principal axes deviates by more than $10^{\circ}$ for both $\mathrm{Cu} 1$ and $\mathrm{Cu} 2$ sites in the opposite directions, which is quite improbable, and would have been detected in previous susceptibility and ESR measurements $[9,12]$.

\section{Nuclear quadrupolar resonance-AFM phase}

Below temperature $T_{N}$, the system enters a magnetically ordered state, and each NQR line splits into four lines without an external field, indicating the existence of the local magnetic field (staggered antiferromagnetic field). In Fig. 5(a) we plot the line positions with respect to temperature. By lowering temperature the staggered field intensity increases thus shifting the lines further apart. The splitting of only $\approx 9 \mathrm{MHz}$ at $1.7 \mathrm{~K}$ is quite small for a copper nucleus that has a relatively high- $\gamma_{n}$ value, so already the raw data indicate that the local magnetic field is either small or/and located perpendicular to the $V_{z z}$ direction for which there should be low value of hyperfine coupling. Hamiltonian in Eq. (3) still governs the frequency dependence, but the magnetic-field $B$ and its angle $\vartheta$ are now determined by spin interactions. We can determine the line position for each temperature with free fitting parameters $B=B_{h f}$ and $\vartheta=\vartheta_{B} . v_{Q}$ is expected to remain constant since it depends only on the crystal electric-field $V_{z z}$, and neutron scattering shows negligible variation of the crystal parameters

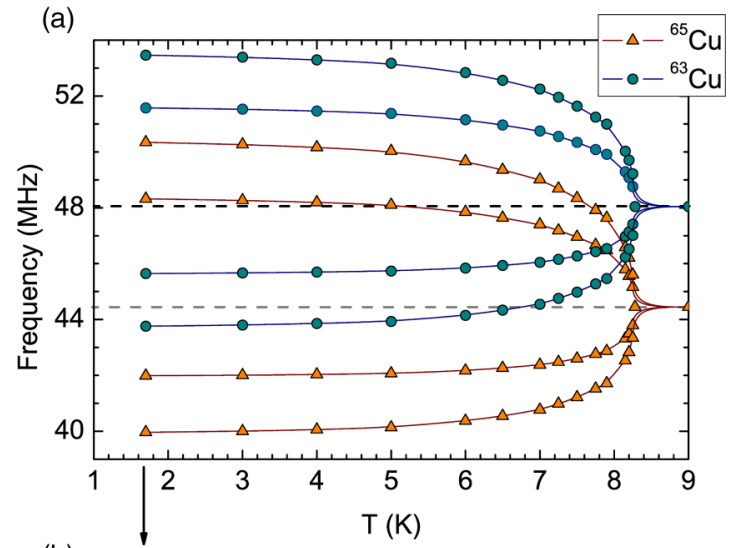

(b)

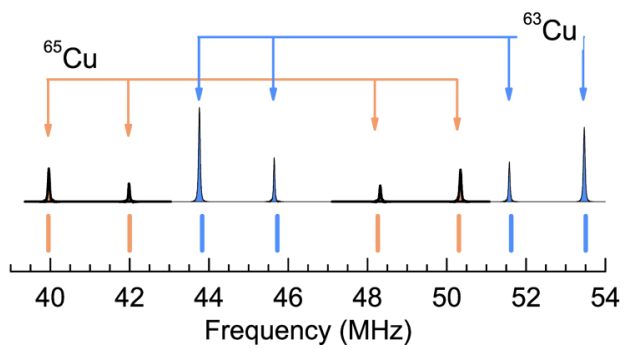

FIG. 5. (a) NQR line positions with respect to temperature. Lines are split because of the staggered magnetic-field $B_{h f}$. As the temperature is lowered, $B_{h f}$ increases monotonously without rotation of the vector in crystal axes (see the text). (b) Line positions of the obtained spectrum at $T=1.7 \mathrm{~K}$ (as indicated by the arrow) with the simulation of the line positions denoted by vertical bars under the spectrum. Parameters are $B_{h f}=0.35 \mathrm{~T}, \vartheta_{B}=80.7^{\circ}$.

in this temperature region. Figure 5(b) presents the NQR fit for the lowest temperature where the local magnetization is completely developed. Here, as anticipated, we obtain $B_{h f}(T=1.7 \mathrm{~K})=0.35 \mathrm{~T}$ and $\theta_{B}=80.7^{\circ}$. This result is quite robust and comes directly from the raw data. In particular, the value of $B_{h f}$ is determined by the magnitude of line splitting for each isotope and the isotope's relative position. On the other hand, the angle of the field $\theta_{B}$ is set by the distribution of the four lines for each isotope. The excellent agreement of the fit shows that the values of $B_{h f}$ and $\theta_{B}$ cannot be varied much. To fit the data at other temperatures it was sufficient to vary only the intensity of magnetic-field $B_{h f}$, whereas the angle $\theta_{B}=80.7^{\circ}$ remained constant. Thus, we can conclude that there is no rotation of the magnetic moment in the ordered state within the sensitivity of our measurements. This is consistent with the previous ESR results which found only a minor rotation (close to $T_{N}$ ) of the net magnetic axes [12]. This is then the consequence of an interplay of the temperature dependence of two noncollinear moments at two copper sites. We note that in Ref. [13] the study of ${ }^{77} \mathrm{Se}$ NMR at a magnetic field close to $10 \mathrm{~T}$ found an anomaly at $6 \mathrm{~K}$ that was ascribed to the spin reorientation. Our measurements are in line with this since the reported [9] spin-flop field in this system is $2 \mathrm{~T}$ for the equivalent orientation of the magnetic field, whereas our low-temperature measurements were performed in a zero magnetic field where no spin reorientation could occur.

To determine the orientation of the magnetization vector $\boldsymbol{m}$ on the $\mathrm{Cu} 1$ site with respect to $V_{z z}$, we need to take into account 


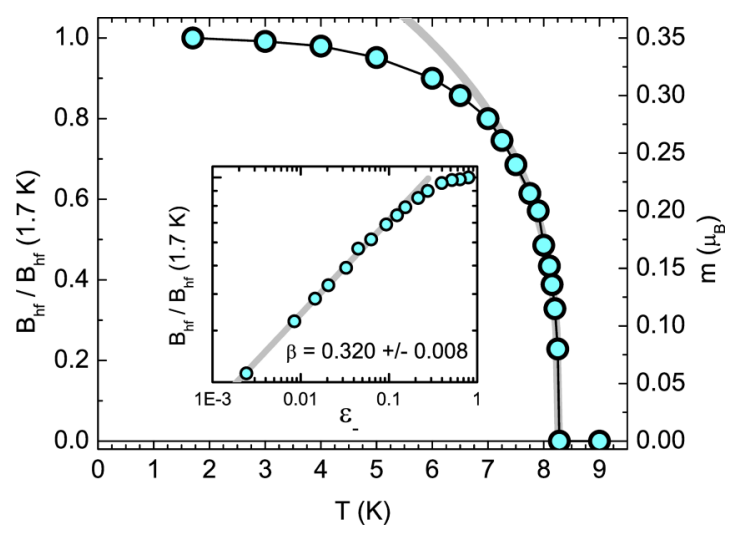

FIG. 6. Staggered field as the order parameter extracted from spectra. The gray line is the fit to expression $5 . B_{h f}(1.7 \mathrm{~K})=0.35 \mathrm{~T}$. The scale on the right shows the corresponding value of magnetization calculated from approximated hyperfine interaction. It is consistent with neutron measurements (see the text). The inset: Logarithmic plot shows power-law behavior.

the hyperfine coupling tensor and relate it to the hyperfine field $B_{h f}$,

$$
\boldsymbol{B}_{h f}=A_{h f} \cdot \boldsymbol{m},
$$

where $A_{h f}$ is the hyperfine tensor. Since the hyperfine tensor is governed by the same crystal symmetries as the EFG tensor, it is justified to expect that their principal axes are the same. For copper in an octahedral environment (with the active $d_{x^{2}-y^{2}}$ orbital), the hyperfine tensor is typically axial with anisotropy of $A_{z z} / A_{x x} \approx 10$. We take the hyperfine tensor values $A_{z z}=$ $-10 \mathrm{~T} / \mu_{B}, A_{x x}=A_{y y}=-1 \mathrm{~T} / \mu_{B}$ because due to the short $T_{2}$ time at high temperatures we were unable to determine it directly. This result is from Ref. [20] on compound $\mathrm{CuGeO}_{3}$, but similar values (with 10\%-20\% uncertainty) can be found in $\mathrm{CuO}$ and other insulators that have $\mathrm{a} \mathrm{Cu}^{2+}$ ion in an octahedral environment (e.g., in azurite [21]). By inverting Eq. (4) and using data obtained from the NQR experiments, we can deduce the value of the magnetic moment residing on the $\mathrm{Cu} 1$ site to be $m=0.35(3) \mu_{B}$, which is within the upper limit extracted from neutron measurements $\left(m=0.5 \mu_{B}\right)$. Our analysis is correct within the $10 \%-20 \%$, which corresponds to the precision of the hyperfine coupling, but nevertheless it shows that the moment size is reduced, renormalized by quantum fluctuations.

Additionally, analysis suggests that the spin on the $\mathrm{Cu} 1$ site lies almost completely inside the $\mathrm{CuO}_{4}$ plaquette (the moment is at an angle of $89^{\circ}$ from $V_{z z}$ )-this is set by the large anisotropy of $A_{h f}$ inherent to the $\mathrm{Cu}^{2+}$ ion and the small splitting by the measured NQR lines. If the angle was smaller it would result in a larger component of the $A_{z z}=-10 \mathrm{~T} / \mu_{B}$ hyperfine field and would be seen as a much larger splitting of the NQR lines.

Since the staggered field $B_{h f}$ is proportional to the order parameter of the AFM phase transition (Fig. 6), we can deduce a critical behavior below the transition $\left(T<T_{N}\right)$ as follows:

$$
\frac{B_{h f}(T)}{B_{h f}(0)}=\left(\frac{T_{N}-T}{T_{N}}\right)^{\beta}=\varepsilon_{-}^{\beta} .
$$

The critical exponent was extracted from a fit in the range of $\varepsilon_{-}=2 \times 10^{-3}-2 \times 10^{-1}$ (inset, Fig. 6). The fitted value is

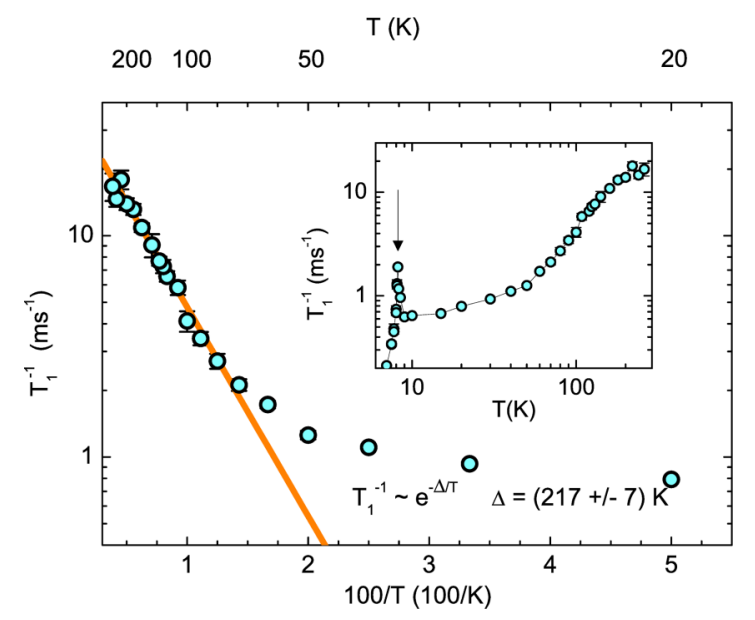

FIG. 7. Arrhenius plot of the spin-lattice relaxation rate: note the inverse temperature and semilogarithmic plot. Points from 90-200 K were fitted. The sharp rise in relaxation with respect to the temperature rise is attributed to breaking the $\mathrm{Cu} 1-\mathrm{Cu} 1$ spin singlet. The inset: Log-log scale. Arrow indicates critical fluctuations at $T_{N}=8 \mathrm{~K}$. Above $200 \mathrm{~K}$ the spin singlet is destroyed which results in a small signal (hence large error bars) and a constant relaxation rate (within experimental error).

shown in Table II together with predictions for several other universality classes. It is obvious that the extracted critical exponent is consistent with the $3 \mathrm{D}$ phase transition. A similar $\beta$ value was found by Lee $e t$ al. in Ref. [13].

\section{Cu1 spin-lattice relaxation}

To get a better insight into the spin dynamics above the ordering temperature, the spin-lattice relaxation rate $\left(1 / T_{1}\right)$ was recorded with the saturation recovery method and is shown in Fig. 7. What can be clearly seen is the activated behavior. Relaxation increases by an order of magnitude from $T=$ 90 to $T=200 \mathrm{~K}$ above which the temperature dependence saturates and $T_{1}$ remains almost constant. This is expected in the paramagnetic state of antiferromagnetic insulators [22] and shows that the truly paramagnetic state exists only at high temperatures. From the Arrhenius plot we can fit the activation energy of $\Delta=217 \pm 7 \mathrm{~K}$, which is close to the $J_{11}$ value determined from the susceptibility [9]. Large error bars in relaxation at high temperatures are caused by the loss of signal due to shortening of spin-spin relaxation time $\left(T_{2}\right)$. The activation behavior indicates that an energy gap opens in the system. Since the ground state of an isolated tetramer system is singlet, the observed temperature dependence most likely originates from a singlet-triplet gap, which means that below 200-K singlets form at the $\mathrm{Cu} 1$ sites (dimer) as was proposed in earlier work [9]. At temperatures lower than $70 \mathrm{~K}$, additional parts of the isolated-tetramer Hamiltonian start to contribute to the relaxation process, and the $1 / T_{1}(T)$ dependence deviates from the activated behavior.

\section{DISCUSSION}

In earlier papers $[9,12]$ it was suggested that the unusual behavior of the spin susceptibility arises from the rotation of 
TABLE II. Critical exponent $\beta$ extracted from temperature dependence of the order parameter below $T_{N}$. Listed are values of $\beta$ for universality classes most suited for this problem.

\begin{tabular}{lcccc}
\hline \hline Experimental (this paper) & \multicolumn{2}{c}{ Theoretical } \\
\hline $\mathrm{SeCuO}_{3}$ & Two-dimensional-Ising & 3D-Ising & 3D-Heisenberg & Mean-field \\
$0.320(8)$ & 0.13 & 0.33 & 0.35 & $1 / 2$ \\
\hline \hline
\end{tabular}

the macroscopic magnetic axes as the temperature is lowered below $200 \mathrm{~K}$. This was additionally supported by the ESR and torque measurements, which mapped the temperature dependence of the magnetic axis shift [12]. The authors hypothesized that this behavior is related to the formation of a singlet state on the inner $\mathrm{Cu} 1-\mathrm{Cu} 1$ pair where the contribution from the $\mathrm{Cu} 1$ site's local axes of anisotropy to the total magnetic anisotropy diminishes and the net magnetization axes rotate towards those defined by the $\mathrm{Cu} 2$ site.

The evidence accumulated from two microscopic techniques presented in this article strongly supports this hypothesis. Additional possibilities included a dynamic Jahn-Teller effect or a structural transition, but the smooth temperature dependence of the NQR line (not shown) excludes this possibility. At high temperatures the $T_{1}(T)$ relaxation data measured at the $\mathrm{Cu} 1$ site show a clear exponential decay that indicates a formation of a singlet-triplet gap. On the other hand, below $T_{N}$, when the system enters an ordered state driven by intertetramer interactions, both neutron diffraction and NQR indicate a greatly reduced value of the ordered magnetic moment residing on the $\mathrm{Cu} 1$ site $(\approx 35 \%$ of fully polarized spin even at $T=1.7 \mathrm{~K}$ ). Even $m_{\mathrm{Cu} 2}$, although less strongly coupled to the central pair, shows its value significantly renormalized $\left(<0.8 \mu_{\mathrm{B}}\right)$. From the low-temperature dependence of the moment on the $\mathrm{Cu} 1$ site, which shows a changing size but stable orientation, we deduce that the rotation of the net magnetization [12] below $T_{N}$ can only appear when the two moments are noncollinear. With this evidence we can establish $\mathrm{SeCuO}_{3}$ as a prime example for further studies of the influence of strong quantum fluctuations on the formation of long-range magnetic order.

The singlet formation at $\mathrm{Cu} 1$ sites is also consistent with magnetization measurements [13] that show magnetization plateau at half the total magnetization, indicating only a $\mathrm{Cu} 2$ spin site contribution to total magnetization. Similar dimerization is reported in the spin-tetramer compound $\mathrm{CuInVO}_{5}$ [10] and in the spin system azurite $\mathrm{Cu}_{3}\left(\mathrm{CO}_{3}\right)_{2}(\mathrm{OH})_{2}$ that has two copper sites with the innermost site experiencing only $10 \%$ of the fully polarized spin [21]. However, only in $\mathrm{SeCuO}_{3}$ the specific magnetic interaction causes such a heterogeneous behavior of the two $\mathrm{Cu}$ moments that results with unusual macroscopic behavior.

We would also like to address the fact that the observed exponential dependence of $T_{1}^{-1}$ is visible down to approximately $60 \mathrm{~K}$ below which it starts to saturate. Around the same tem- perature a simple tetramer model of susceptibility breaks down [9]. This shows that some other energy scale starts contributing to $T_{1}$, most probably the anisotropic Dzyaloshinskii-Moriya (DM) interaction. The deviation from activated behavior so high in temperature cannot originate from critical fluctuations of AF order since it onsets only below $T_{N}=8 \mathrm{~K}$, and the increase in $1 / T_{1}$ is visible only $1 \mathrm{~K}$ above $T_{N}$. The influence of $\mathrm{DM}$ interaction is in agreement with the additional ESR data [12] where the rotation of the magnetic axis below $\approx 50 \mathrm{~K}$ was ascribed to the $\mathrm{DM}$ interaction between $\mathrm{Cu} 1$ and $\mathrm{Cu} 2$. Lack of the $\mathrm{Cu} 2$ signal in NQR can then be explained as $\mathrm{Cu} 2$ coupling is weaker and acts as an almost free spin with strong magnetic fluctuations that cause short $T_{2}$ time. On the other hand, the spin singlet on $\mathrm{Cu} 1$ greatly increases the relaxation time thus making that site visible in magnetic resonance experiments.

To summarize, we have presented microscopic evidence by combining neutron diffraction with NQR measurements that supports the hypothesis of $\mathrm{SeCuO}_{3}$ being formed of weakly coupled magnetic tetramers in the presence of the Dzyaloshinskii-Moriya interaction. The central pair of spins, the $\mathrm{Cu} 1-\mathrm{Cu} 1$ pair, is strongly coupled and forms a spin singlet state below $T<J_{11}$, whereas the $\mathrm{Cu} 2$ spins remain weakly coupled to the central pair. Both neutron and NQR measurements detected an unexpectedly low-ordered moment on the $\mathrm{Cu} 1$ site, renormalized by quantum fluctuations.

\section{ACKNOWLEDGMENTS}

T.C. and M.S.G. are thankful to M. Horvatić, X. Rocquefelte, and W. Lafargue-dit-Hauret for a thorough discussion of the results. T.C., M.S.G., and M.P. acknowledge the Croatian Science Foundation (HRZZ) for funding the NMR/NQR studies under Grant No. IP-11-2013-2729. Financial support from the Swiss National Science Foundation (SNSF) Project Grants No. 169699 and No. 166298 is gratefully acknowledged. Neutron experiments have been carried out within the framework of the EUROfusion Consortium, and funding has been received from the Euratom Research and Training Programme 2014-2018 under Grant Agreement No. 633053. The views and opinions expressed herein do not necessarily reflect those of the European Commission. Neutron-diffraction experiments have been carried out at SINQ PSI, Villigen, Switzerland.

T.C. and V.S. contributed equally to this work.
[1] Y. Li, D. Adroja, P. K. Biswas, P. J. Baker, Q. Zhang, J. Liu, A. A. Tsirlin, P. Gegenwart, and Q. Zhang, Phys. Rev. Lett. 117, 097201 (2016).
[2] T.-H. Han, J. S. Helton, S. Chu, D. G. Nocera, J. A. RodriguezRivera, C. Broholm, and Y. S. Lee, Nature (London) 492, 406 (2012). 
[3] R. Sessoli, D. Gatteschi, A. Caneschi, and M. A. Novak, Nature (London) 365, 141 (1993).

[4] C. Rüegg, A. Furrer, D. Sheptyakov, T. Strässle, K. W. Krämer, H.-U. Güdel, and L. Mélési, Phys. Rev. Lett. 93, 257201 (2004).

[5] J. T. Haraldsen, T. Barnes, and J. L. Musfeldt, Phys. Rev. B 71, 064403 (2005).

[6] M. Prester, A. Smontara, I. Živković, A. Bilušić, D. Drobac, H. Berger, and F. Bussy, Phys. Rev. B 69, 180401(R) (2004).

[7] C. N. Kuo and C. S. Lue, Phys. Rev. B 78, 212407 (2008).

[8] M. Hase, M. Kohno, H. Kitazawa, O. Suzuki, K. Ozawa, G. Kido, M. Imai, and X. Hu, Phys. Rev. B 72, 172412 (2005).

[9] I. Živković, D. M. Djokić, M. Herak, D. Pajić, K. Prša, P. Pattison, D. Dominko, Z. Micković, D. Cinčić, L. Forró, H. Berger, and H. M. Rønnow, Phys. Rev. B 86, 054405 (2012).

[10] M. Hase, M. Matsumoto, A. Matsuo, and K. Kindo, Phys. Rev. B 94, 174421 (2016).

[11] H. Effenberger, Z. Kristallogr. 175, 61 (1986).

[12] M. Herak, A. Grubišić Čabo, D. Žilić, B. Rakvin, K. Salamon, O. Milat, and H. Berger, Phys. Rev. B 89, 184411 (2014).
[13] S. Lee, W.-J. Lee, J. van Tol, P. L. Kuhns, A. P. Reyes, H. Berger, and K.-Y. Choi, Phys. Rev. B 95, 054405 (2017).

[14] J. Rodriguez-Carvajal, Physica B (Amsterdam) 192, 55 (1993).

[15] A single-crystal diffraction experiment has been also performed in order to provide more details about the structure. Unfortunately, the resulting dataset proved to be unreliable.

[16] C. P. Slichter, Principles of Magnetic Resonance, 3rd ed. (Springer-Verlag, Berlin, New York, 1990), Chap. 10.

[17] I. Kupčić, S. Barišić, and E. Tutis, Phys. Rev. B 57, 8590 (1998).

[18] E. N. Kaufmann and R. J. Vianden, Rev. Mod. Phys. 51, 161 (1979).

[19] The magic angle is a root of the second-order Legendre polynomial $P_{2}\left(\cos \vartheta_{m}\right)=0, \vartheta_{m}=54.7^{\circ}$. At this angle first-order quadrupolar interaction vanishes, i.e., two inner NQR lines merge.

[20] M. Itoh, S. Hirashima, and K. Motoya, Phys. Rev. B 52, 3410 (1995).

[21] F. Aimo, S. Krämer, M. Klanjšek, M. Horvatić, C. Berthier, and H. Kikuchi, Phys. Rev. Lett. 102, 127205 (2009).

[22] T. Moriya, Prog. Theor. Phys. 16, 23 (1956). 